\title{
Liquid sloshing in the horizontal circular tanks with complex baffles
}

\author{
Jun Liu ${ }^{1, a}$, Pengchong Zhang ${ }^{1, b}$ \\ ${ }^{1}$ School of Hydraulic Engineering, Faculty of Infrastructure Engineering, Dalian University of \\ Technology, Dalian 116024,China \\ aliujun8128@126.com, bhangpengchong2014@126.com
}

\begin{abstract}
Keywords: Sloshing, Scaled boundary finite element method, Circular tanks, Baffles, Sloshing frequencies.
\end{abstract}

Abstract. A semi-analytical hydrodynamic analysis based on the linear potential theory is introduced to study the liquid sloshing in horizontal circular cylindrical T-shaped baffled container using the novel scaled boundary finite element method (SBFEM). Via variational principle formulation, a system of ordinary differential equations for the determination of the unknown potential functions for the sloshing problem is obtained, which can be solved in a closed-form analytical manner. Moreover, another innovative solution for the inclusion of flow velocity along the baffles, which is called the side-faces of bounded domain in SBFEM, can also be analytically obtained. Two special T-shaped baffled configurations are considered including surface-piercing baffle and bottom-mounted baffle. Numerical examples show the accuracy and efficiency of the pro-posed method applied to the sloshing problems with baffles. The effects of parameters of baffles on the sloshing frequencies, the associated sloshing mode shapes and sloshing wave height are examined in details.

\section{Introduction}

Liquid sloshing is very important in a wide range of engineering such as the safety of transportation systems, human's life and environment on account of economically and commercially valuable liquids such as ships, satellites, rockets, trucks and even stationary petroleum containers [1], which might cause catastrophic economical and human-life losses as well as environmental damages. Several passive means are adopted in practice to overcome such undesirable effects. One of the passive devices to control liquid flow and suppress liquid sloshing is the baffle installed inside a tank, and it has attracted the attention of many researchers.

The issue of suppression of sloshing behavior using baffles goes back to late 50s when lots of experimental and theoretical studies were conducted for the effect of baffles on the sloshing in fuel containers of space vehicles [2]. There has been many research works in the literature dealing with sloshing problems with baffles, which can be performed using experimental models or analytical approaches techniques [3,4]. With the rapid development of the computer performance over half a century, computational methods have become robust tool for many field analyses of sloshing problems. Among them, the finite element method [5], boundary element method [6, 7] have become many successful uses in numerical analysis of sloshing with baffles. Among them, the Finite ele-ment method (FEM) is one of the undoubtedly dominant methods for modeling the sloshing problems with different baffles, because of its powerful capability of simulating a large variety of problems with complex geometries and various boundaries. However, in applying the finite element method to sloshing problems, the entire liquid volume has to be discretized, which calls for relatively large computing resources. The boundary element method where only the liquid boundary, rather than the entire volume, needs to be discretized offers an efficient alternative to the finite element method. But, difficulties are also encountered in applying the boundary element method to many complex sloshing problems with different baffles owing to its reliance on the fundamental solution, and fundamental solution does not exit in some cases. Meanwhile, according to the authors' literature survey, simulation of liquid sloshing in a circular container with T-shaped or Y-shaped baffles seems to be non-existent. The purpose of this paper is to provide a simple and new semi-analytical method, which is named scaled boundary finite element (SBFEM), to simulate the sloshing problems with baffles of 
complex geometry. The SBFEM originally has been developed to solve soil-structure interaction problems, which was proposed [8] and systematically described [9] by Wolf and Song. The SBFEM combines the advantages of FEM and BEM, i.e., it reduces the modelled spatial dimensions by one and discretises boundaries only as the BEM, and mean-while, it does not need fundamental solutions as the FEM.

\section{Theoretical description}

Fig.1 shows the schematic diagram of an inviscid incompressible liquid containing two-dimensional circular tank with vertical T-shaped baffles, which is subjected to forced horizontal excitation. Where, $R$ is the length of circular radius, and $h$ is the water depth. Three typical T-shaped baffle configurations are investigated including surface-piercing baffle, bottom-mounted baffle and their combination form. In Fig. $1,2 L_{1}$ and $L_{2}$ represent the horizontal and vertical length of the T-shaped baffles, respectively, and $2 d$ is corresponding to the width of the water surface in the tank. The governing equation of liquid motion is described, which satisfies the Laplace equation and the associated boundary conditions (Fig. 1) expressed as

$$
\frac{\partial^{2} \varphi}{\partial x^{2}}+\frac{\partial^{2} \varphi}{\partial y^{2}}=0 \quad \text { in } \Omega
$$

Based on linear potential theory, the corresponding boundary conditions are as follow

$$
\begin{array}{cl}
\text { at the wet surface of the tank wall } B_{1} & \frac{\partial \varphi}{\partial n}=v_{n} \\
\text { and at the free surface } B_{2} & \frac{\partial \varphi}{\partial y}=\frac{\omega^{2}}{g} \varphi
\end{array}
$$

where $\mathrm{g}$ is the gravitational acceleration, $v_{n}$ is the vector of exciting velocity, and $n$ is its normal component at the tank wall.

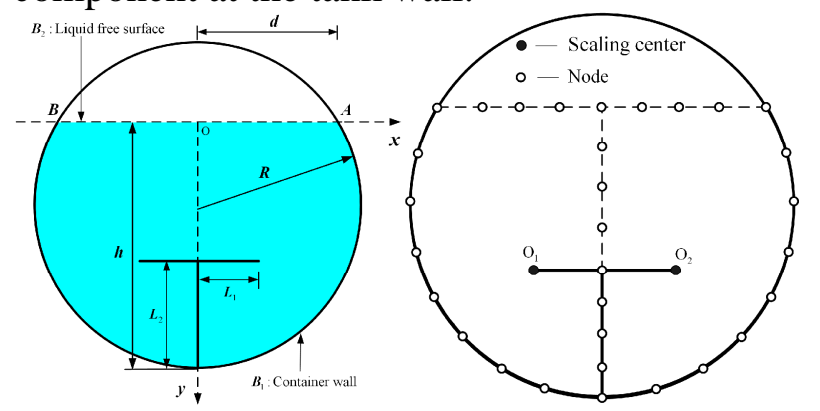

(a) A bottom-mounted vertical baffle
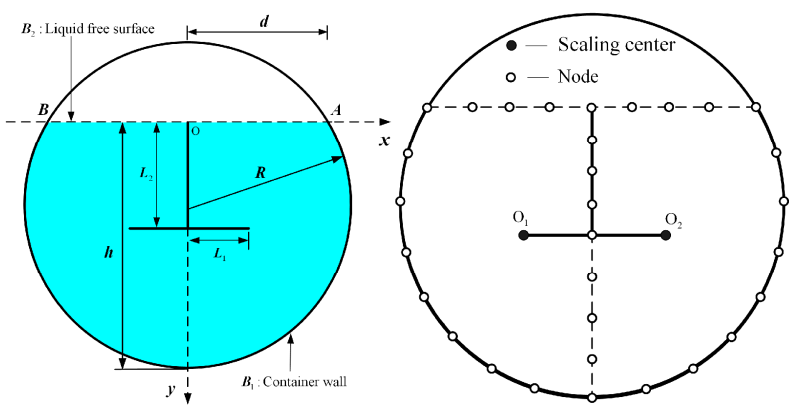

(b) A surface-piercing vertical baffle

Figure 1. Schematic and SBFEM mesh of baffled circular containers.

\section{Scaled boundary finite element method for the problem analysis}

This problem, which combines the governing Eq. (1) and boundary conditions Eqs. (2) and (3) indirectly via the equivalent variational problem, can be defined by

$$
\delta F(\varphi)=\delta\left(\frac{1}{2} \int_{\Omega}\left(\frac{\partial \varphi}{\partial x}\right)^{2}+\left(\frac{\partial \varphi}{\partial y}\right)^{2} d \Omega-\int_{\Gamma} \psi_{n} d \Gamma\right)=0
$$

where the right parts of Eqs. (2) and (3) are unified into a form $v_{n}$. 


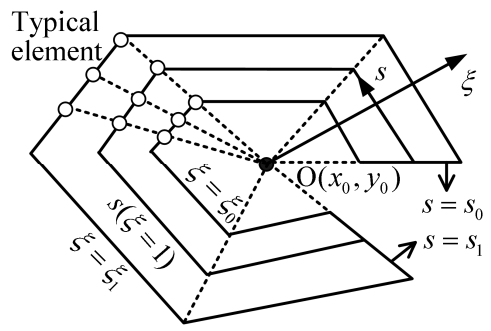

Figure 2. The coordinate definition of SBFEM.

To apply the SBFEM to Eq. (6), the so-called scaled boundary coordinate system is introduced. As shown in Fig.2, a domain is formed by scaling a defining curve $S$ in the radial direction with respect to the centre $O\left(x_{0}, y_{0}\right)$ from which the entire boundary is visible. The SBFEM introduces two coordinates, Namely, the radial coordinate $\xi$ and the circumferential coordinate $s$. $\xi$ points to the boundary, which is a scaling factor and is in the range of $0=\xi_{0} \leq \xi \leq \xi_{1}=1$, and $s$ is along the boundary direction. The transformation equation relating between this coordinate system $(\xi, s)$ and the Cartesian coordinate system $(x, y)$ can be expressed

$$
x=x_{0}+\xi x(s) ; \quad y=y_{0}+\xi y(s)
$$

Proceeding in the common derivation of SBFEM equation which can be referred to the literature (Wolf and Song, 2003), the SBFE equation in sloshing potential is expressed as

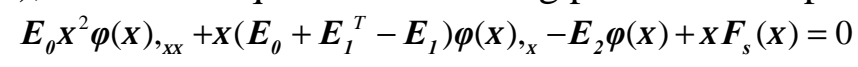

and the associated boundary condition

$$
\begin{gathered}
\boldsymbol{E}_{0} \xi \boldsymbol{\varphi}(\xi)_{,_{\xi}}+\left.\boldsymbol{E}_{I}^{T} \boldsymbol{\varphi}(\xi)\right|_{\xi=\xi_{1}}=\int_{s} \boldsymbol{N}(s)^{T} v_{n} d \Gamma \\
\boldsymbol{E}_{0} \xi \boldsymbol{\varphi}(\xi)_{,_{\xi}}+\left.\boldsymbol{E}_{l}^{T} \boldsymbol{\varphi}(\xi)\right|_{\xi=\xi_{1}}=\frac{\omega^{2}}{g} \boldsymbol{M}_{0} \boldsymbol{\varphi}(\xi)
\end{gathered}
$$

where $\boldsymbol{E}_{\boldsymbol{0}}, \boldsymbol{E}_{1}, \boldsymbol{E}_{2}$ and $\boldsymbol{M}_{\boldsymbol{0}}$ are coefficient matrices calculated in relation with the discretization of the domain boundary, and the non-homogeneous term $\boldsymbol{F}_{s}(\xi)$ is due to the prescribed exciting velocity of the side-faces.

To transform Eq. (6) into a simpler ordinary differential equation, a variable is introduced

$$
\boldsymbol{X}(\xi)=\left\{\begin{array}{l}
\boldsymbol{\varphi}(\xi) \\
\boldsymbol{Q}(\xi)
\end{array}\right\}, \text { where } \boldsymbol{Q}(\xi)=\boldsymbol{E}_{0} \xi \boldsymbol{\varphi}(\xi)_{,}+\boldsymbol{E}_{1}^{T} \boldsymbol{\varphi}(\xi)
$$

Substituting Eq. (9) into Eq. (6) yields

$$
\xi \boldsymbol{X}(\xi)_{,_{\xi}}=-\boldsymbol{Z X}(\xi)-\left\{\begin{array}{c}
0 \\
\boldsymbol{F}(\xi)
\end{array}\right\} \text {, where } \boldsymbol{Z}=\left[\begin{array}{cc}
\boldsymbol{E}_{0}^{-1} \boldsymbol{E}_{1}^{T} & -\boldsymbol{E}_{0}^{-1} \\
-\boldsymbol{E}_{2}+\boldsymbol{E}_{1} \boldsymbol{E}_{0}^{-1} \boldsymbol{E}_{1}^{T} & -\boldsymbol{E}_{1} \boldsymbol{E}_{0}^{-1}
\end{array}\right]
$$

The following eigenvalue problem for the homogeneous governing equation (10) is solved ( $\Phi$ is corresponding to the eigenvector.)

$$
Z \Phi=-\Phi \Lambda
$$

Then, the solution of Eq.(10) can be obtained as

$$
\left(\left[\begin{array}{cc}
\boldsymbol{K}_{a a} & \boldsymbol{K}_{a b} \\
\boldsymbol{K}_{b a} & \boldsymbol{K}_{b b}
\end{array}\right]-\frac{\omega^{2}}{g}\left[\begin{array}{cc}
\boldsymbol{M}_{0} & 0 \\
0 & 0
\end{array}\right]\right)\left\{\begin{array}{l}
\boldsymbol{\varphi}_{a} \\
\boldsymbol{\varphi}_{b}
\end{array}\right\}=\left\{\begin{array}{c}
0 \\
\boldsymbol{Q}_{b}
\end{array}\right\}+\boldsymbol{R}^{F}
$$

where

$$
\boldsymbol{R}^{F}=\boldsymbol{A}_{22}^{-1} \int_{0}^{1}\left\lceil\tau^{-\lambda_{i}-1}\right\rfloor \boldsymbol{A}_{22} \boldsymbol{F}(\tau) d \tau, \quad \text { in which } \boldsymbol{A}=\left[\begin{array}{ll}
\boldsymbol{A}_{11} & \boldsymbol{A}_{12} \\
\boldsymbol{A}_{21} & \boldsymbol{A}_{22}
\end{array}\right]=\left[\begin{array}{ll}
\boldsymbol{\Phi}_{11} & \boldsymbol{\Phi}_{12} \\
\boldsymbol{\Phi}_{21} & \boldsymbol{\Phi}_{22}
\end{array}\right]^{-1}=\boldsymbol{\Phi}^{-1}
$$




\section{Validation of SBFEM model and numerical results}
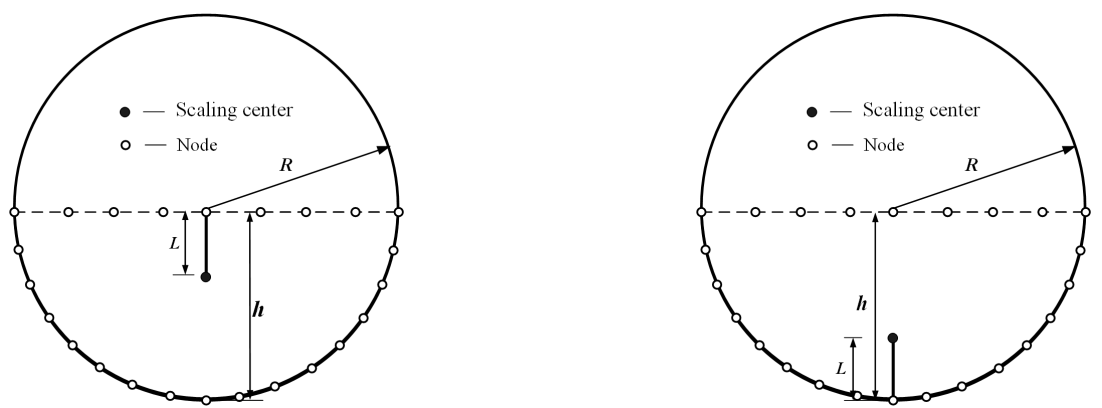

Figure 3. SBFEM mesh of baffled half-full circular containers with (a) a surface-piercing vertical baffle, and (b) a bottom-mounted vertical baffle.

Table 1. Comparison of the calculated normalized frequencies of slosh in a baffled half-full circular container with those of literature.

\begin{tabular}{|c|c|c|c|c|c|c|c|c|c|}
\hline \multicolumn{4}{|c|}{ surface-piercing vertical baffle } & \multicolumn{5}{|c|}{ bottom-mounted vertical baffle } \\
\hline Mode & $\begin{array}{c}\text { Hasheminejad } \\
\text { and } \\
\text { Mohammadi }\end{array}$ & $\begin{array}{c}\text { SBFEM } \\
(16 \\
\text { elements) }\end{array}$ & $\begin{array}{c}\text { SBFEM } \\
(32 \\
\text { elements })\end{array}$ & $\begin{array}{c}\text { SBFEM } \\
(64 \\
\text { elements })\end{array}$ & Mode & $\begin{array}{c}\text { Hasheminejad } \\
\text { and } \\
\text { Mohammadi }\end{array}$ & $\begin{array}{c}\text { SBFEM } \\
(16 \\
\text { elements) }\end{array}$ & $\begin{array}{c}\text { SBFEM } \\
(32 \\
\text { elements })\end{array}$ & $\begin{array}{c}\text { SBFEM } \\
(64 \\
\text { elements })\end{array}$ \\
\hline 1 & 0.8511 & 0.8511 & 0.8507 & 0.8501 & 1 & 1.2171 & 1.2182 & 1.2157 & 1.2126 \\
\hline 2 & 3.0339 & 3.0362 & 3.0342 & 3.0312 & 2 & 3.0334 & 3.0358 & 3.0328 & 3.0312 \\
\hline 3 & 3.1370 & 3.1465 & 3.1365 & 3.1345 & 3 & 4.6341 & 4.6431 & 4.6342 & 4.6319 \\
\hline 4 & 6.2416 & 6.2508 & 6.2413 & 6.2401 & 4 & 6.2414 & 6.2435 & 6.2411 & 6.2399 \\
\hline 5 & 6.2431 & 6.2533 & 6.2428 & 6.2420 & 5 & 7.8200 & 7.9068 & 7.8193 & 7.8174 \\
\hline $\begin{array}{c}\text { Times } \\
\text { (s) }\end{array}$ & - & 0.08 & 0.23 & 0.92 & $\begin{array}{c}\text { Times } \\
(\mathrm{s})\end{array}$ & - & 0.07 & 0.25 & 0.95 \\
\hline
\end{tabular}

In order to verify the correctness and efficiency of the proposed method, a horizontal cylindrical container of circular cross section fitted with vertical baffles is provided as test cases and the results have been compared with the limit literature (Hasheminejad and Mohammadi, 2011) [10]. The three-nodes quadratic SBFEM element is used, which is also used in all this paper. Three meshes are used to illustrate the convergence of the method, as shown in Fig.3. The coarse mesh consists of 16 elements with 33 nodes, as illustrated in Fig.3. The other two meshes (referred to as intermediate and fine) are constructed by binary subdivision of the coarse mesh, and consist of 65 and 129 nodes respectively. Meanwhile, the scaling center is placed at the tip of the baffle, in which boundary of the baffle can not discretized and the singularity at the tip can be easily simulated. The normalized first five natural slosh frequencies $\Omega=\omega^{2} R / g$, which excludes the zero frequency corresponding to the rigid body mode, are compared with those reported by Hasheminejad and Mohammadi (2011) [10] in Table 1 , in which $L=0.5 R$. The results with only 16 elements agree well with those reported from the solutions in Reference [10]. The CPU computation times (an Intel Core(TM) i7 platform at $3.6 \mathrm{GHz}$ with 16.0 GB RAM) for the SBFEM are also given in table 1. From the table, this proposed method can achieve excellent accuracy and convergence, and have higher efficiency with significantly fewer nodes and much less consumed time.

In this section, a parametric study is carried out to investigate the effects of the key geometric parameters, namely the liquid fill level and the location, vertical length and horizontal length of the T-shaped baffles, on the natural sloshing frequencies, mode shapes and sloshing wave height. Two cases with the liquid fill level $h=1.7071 R$ ( $x$ coordinate of point A in Fig. 3 is equal to $\left.R \sin \left(45^{\circ}\right)\right)$ and $h=R$ ( $x$ coordinate of point A in Fig. 1 is equal to $\left.R \sin \left(0^{0}\right)\right)$ are mainly considered. The tank is assumed to be oscillating in the horizontal direction with amplitude $A$ and an angular frequency $\omega$, and then the velocity of the tank motion is written as $u=\omega A \cos (\omega t)$. Two exciting velocity of the tank are considered including $\omega /(g / R)^{1 / 2}=1,2$. In the SBFEM, only the boundary of the fluid domain is discretized into boundary finite elements with three-node second-order finite elements. The meshes of horizontal circular tanks with surface-piercing baffle, bottom-mounted baffle and their combination form are shown in Fig.1, in which the scaling centers are all placed at the tip of the baffles, and there are two sub-structures constituting 159 nodes of SBFEM for the tanks with surface-piercing baffle 
and bottom-mounted baffle as shown in Figs.1(a) and (b).Fig.4 depicts the wave surface elevation along the water surface level at the moment $\omega t=2 n \pi(n=0,1,2, \mathrm{~L})$ with different vertical length ratio $L_{2} / h$. It can be observed from Fig. 5 that, in generally, when the baffles are not near to the liquid free surface, the vertical length $L_{2}$ has a little effect on the wave surface elevation, especially for the high fill levers. Surface-piercing baffles may bring our higher height of the liquid than those of combination form baffles. In the case of surface-piercing and combination form baffles with lower fill levers such as $h=R$, the curves of wave surface elevation excited by $\omega /(g / R)^{1 / 2}=1$ bring out not the linear change along the water surface level compared to those of the other cases.

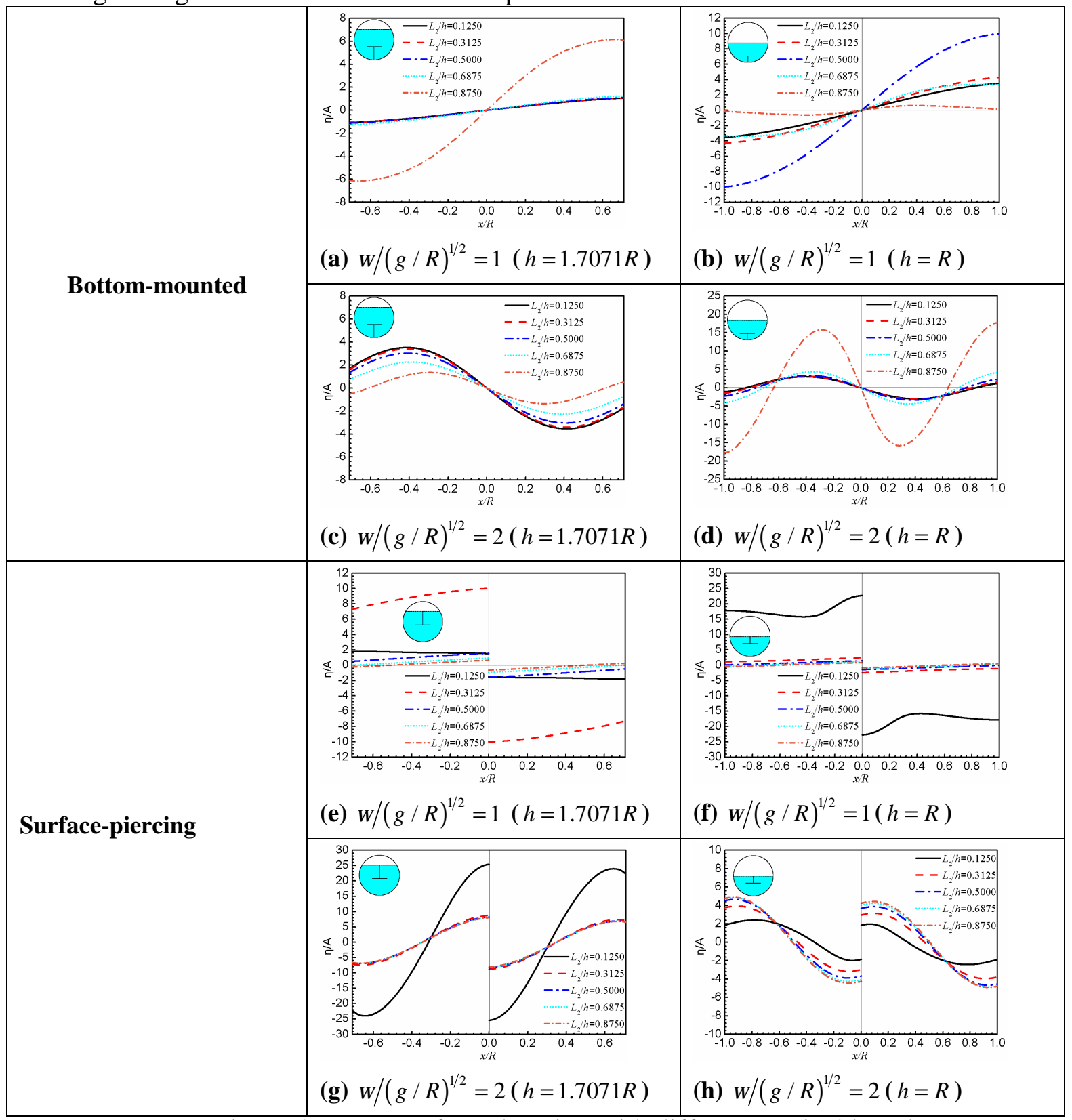

Figure 4. Water surface elevation with different vertical length.

\section{Conclusions}

A semi-analytic approach named scaled boundary finite element method based on the linearized theory of surface gravity waves is employed to investigate the transverse two-dimensional sloshing problems of a perfect liquid partially filling a non-deformable horizontal circular cylindrical container, fitted with surface-piercing ,bottom-mounted vertical T-shaped baffle of arbitrary extension. The proposed method can reduce one in the spatial dimensions, thus only the boundary of tanks is 
discretized with surface finite elements. The detailed derivation and solution for the sloshing problems based on the scaled boundary finite element method. Meanwhile, Analytical solution for non-homogeneous term including inclusion of flow velocity along the baffles is derived, and the eigenvalue problems for free liquid sloshing and the hydrodynamic equation of liquid sloshing for SBFEM are also obtained. The numerical results validate that the proposed method can achieve excellent accuracy, and it has higher convergence and efficiency with significantly fewer nodes and much less consumed time. A detailed parametric study for examining the influence of the container's baffled arrangement on the first few calculated liquid natural frequencies and water surface elevation is performed.

\section{Acknowledgements}

This research was supported by Grants 51409038, and Grant DUT15RC(4)23 from the fundamental research funds for the central universities for which the authors are grateful.

\section{References}

[1] Ibrahim, R.A.. (2005) Liquid Sloshing Dynamics. Cambridge University Press, Cambridge.

[2] Abramson, H.n.(1969) Slosh suppression. NASA Technical Report SP-8031.

[3] Maleki, A., Ziyaeifar, M.(2008) Sloshing damping in cylindrical liquid storage tanks with baffles. Journal of Sound and Vibration, 311, 372-385.

[4] Akyıldız, H., Ünal N. E., Aksoy H.(2013) An experimental investigation of the effects of the ring baffles on liquid sloshing in a rigid cylindrical tank. Ocean Engineering, 59,190-197.

[5] Biswal, K.C., Bhattacharyya, S.K., Sinha P.K.(2004) Dynamic response analysis of a liquid-filled cylindrical tank with annular baffle. Journal of Sound and Vibration, 274, 1-2, 13-37.

[6] Ebrahimian, M., Noorian M.A., Haddadpour H.(2013) A successive boundary element model for investigation of sloshing frequencies in axisymmetric multi baffled containers. Engineering Analysis with Boundary Elements, 37,383-392.

[7] Ebrahimian, M., Noorian M.A., Haddadpour H.(2014) Equivalent mechanical model of liquid sloshing in multi-baffled containers. Engineering Analysis with Boundary Elements,47,82-95.

[8] Song, C. M., Wolf, J. P.(1997) The scaled boundary finite-element method-Alias consistent infinitesimal finite-element cell method-for elastodynamics. Computer Methods in Applied Mechanics and Engineering, 147,329-355.

[9] Wolf, J. P., Song, C. M.(2003) The scaled boundary finite element method, in Chichester: England, Wiley Press.

[10] Hasheminejad, S. M., Mohammadi, M. M.(2011) Effect of anti-slosh baffles on free liquid oscillations in partially filled horizontal circular tanks. Ocean Engineering, 38, 49-62. 